See Article page 1030.

\section{Commentary: Cardiothoracic surgery may be more like special ops than commercial aviation}

\author{
J. W. Awori Hayanga, MD, MPH, MHL, \\ Jason Lamb, MD, and Chris Cook, MD
}

Feindel and colleagues ${ }^{1}$ elaborate on a spectrum of surveillance to accompany individual surgeon evaluation. It is almost impossible to overemphasize the influence of surgical teams within the system in acknowledgment that surgery is indeed the quintessential team sport. As such, the individual decline of any surgeon may be significantly mitigated by the strengths and protections proffered by the team and the broader processes at work that maintain patient safety and quality. The analogy to the aviation industry is important and relevant. Still, as we draw parallels, we should we not push beyond mere endorsement of surveillance and elaborate instead upon the quality, consistency, and manner of the very surveillance itself. There is a high premium placed on real-time trend surveillance in aviation that may not necessarily find equivalent parallels within surgical environments. ${ }^{2}$ The analogy may derive greater potency by drawing comparisons with military aviation, where early warnings are used to alter strategic command in real time. In this manner, the communion of stakeholders is allowed to make expeditious modifications in operational planning with the explicit purpose of minimizing casualties with as little lag time as possible.

Surveillance systems should create a comprehensive culture of safety beyond simple checklists for hardware and evaluate how long pilots have been in the air at any given time and define limits as to duration of time spent in flight and indeed in active flight duty. ${ }^{2-4}$ Beyond the

\footnotetext{
From the Department of Cardiovascular and Thoracic Surgery, WVU Heart and Vascular Institute, West Virginia University, Morgantown, WVa.

Disclosures: The authors reported no conflicts of interest.

The Journal policy requires editors and reviewers to disclose conflicts of interest and to decline handling or reviewing manuscripts for which they may have a conflict of interest. The editors and reviewers of this article have no conflicts of interest.

Received for publication Nov 12, 2021; revisions received Nov 12, 2021; accepted for publication Nov 17, 2021; available ahead of print Nov 20, 2021.

Address for reprints: J. W. Awori Hayanga, MD, MPH, MHL, Department of Cardiovascular and Thoracic Surgery, WVU Heart and Vascular Institute, West Virginia University, 1 Medical Center Dr, Morgantown, WV 26506 (E-mail: jeremiah. hayanga@wvumedicine.org).

J Thorac Cardiovasc Surg 2022;164:1033-4

$0022-5223 / \$ 36.00$

Copyright (c) 2021 by The American Association for Thoracic Surgery

https://doi.org/10.1016/j.jtcvs.2021.11.040
}

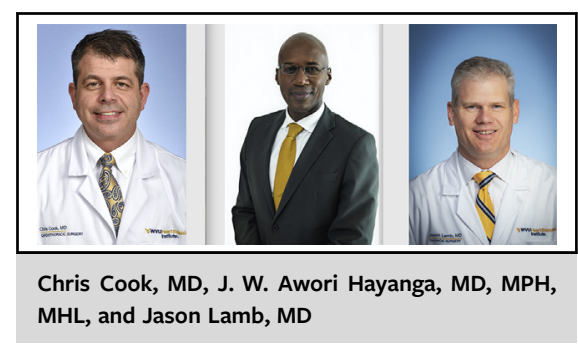

\section{CENTRAL MESSAGE \\ A surgeon's evolution persists long after surgical training and the 6 competencies should continue to influence this evolu- tion and its adjudication.}

endorsement of simulation, the analogy should address that contemporary surgical practice features an absence of criteria to determine how long a surgeon has worked over a period or the quantification of the cumulative burden of chronic fatigue, frequency of calls, and general expectations of hospital administration and patient alike. As such, although the aviation industry provides a convenient analogy, surgical operations in cardiothoracic surgery may be closer akin to military aviation or perhaps part 135 flight operations, which deal with emergencies and are structured somewhat differently from commercial passenger aviation. ${ }^{4}$

The use of simulation has frequently been deemed a useful adjunct in the process of evaluation and maintenance of surgical skill. The fidelity and appropriateness of simulation is difficult to guarantee and the cost of the necessary technology is often prohibitive. Indeed, despite the frequent analogy with crew resource management, surgery often lags behind aviation in many respects and the latter proffers a far greater number safety checks for both pilot and passenger than surgery does for surgeon and patient.

Indeed, it may be an oversimplification to believe that surgical evaluation rests solely on technical considerations when indeed the Accreditation Council for Graduate Medical Education proffers 6 core competencies upon which graduate education is founded, namely patient medical knowledge, systems-based practice, professionalism, practice-based learning, and improvement and interpersonal and communication skills. ${ }^{5}$ The evolution of the surgeon persists long after surgical training and the 6 
competencies should likely, in the same vein, continue to influence not just this evolution but its adjudication as well.

\section{References}

1. Feindel C. Intermittent surgeon career evaluation is needed from beginning to end. J Thorac Cardiovasc Surg. 2022;164:1030-2.

2. Meynard J, Chaudet H, Texier G, Dupuy B, Queyriaux B, Pellegrin L, et al. Advantages and limits of real-time epidemiological surveillance during military deployments: the experience of the French Armed Forces. Mil Med. 2009;174: 1068-74.

3. List of accidents and incidents involving the Boeing 737; 2021. Accessed November 6, 2021. https://en.wikipedia.org/w/index.php?title=List_of_accidents_ and_incidents_involving_the_Boeing_737\&oldid=1021020739

4. Aireon - Space-based ADS-B global air traffic surveillance and tracking. Accessed November 6, 2021. https://aireon.com/

5. Accreditation Council for Graduate Medical Education 202. Accessed November 6, 2021. https://en.wikipedia.org/wiki/List_of_accidents_and_incidents_involving_ commercial_aircraft 